\title{
Physicians' Roles in Creating Health Literate Organizations: A Call to Action
}

\author{
Cindy Brach, MPP ${ }^{7}$, Benard P. Dreyer, $M D^{2}$, and Dean Schillinger, $M D^{3}$
}

'Agency for Healthcare Research and Quality, Rockville, MD, USA; ${ }^{2}$ Department of Pediatrics, New York University, Bellevue Hospital, New York, NY, USA; ${ }^{3}$ Division of General Internal Medicine, Department of Medicine, University of California San Francisco, San Francisco, CA, USA.

J Gen Intern Med 29(2):273-5

DOI: $10.1007 / \mathrm{s} 11606-013-2619-6$

(C) Society of General Internal Medicine 2013

$\mathrm{F}$ ueled by the Affordable Care Act (ACA) and accreditation and credentialing requirements, physicians are being called on to deliver patient-centered care, reduce medical errors and generally increase health care quality and health outcomes, all while containing costs. Health care leaders recognize that fully engaging patients in prevention, decision-making and self-management activities is critical to achieving these aims and that patient engagement entails addressing health literacy.

Health literacy is defined by the ACA as "the degree to which an individual has the capacity to obtain, communicate, process, and understand health information and services in order to make appropriate health decisions." That capacity is determined not only by individuals' skills and abilities, but also by the complexities of the health care system that can increase the communication demands placed on patients. With only $12 \%$ of American adults having health literacy skills proficient enough to successfully understand health information and navigate our demanding health care system, ${ }^{1}$ revamping how health information and health care are delivered has to be a priority.

The National Action Plan to Improve Health Literacy of $2010^{2}$ set the stage for widespread adoption of health literacy goals. It calls for both organizations and professionals to participate in a multi-sector effort to improve health literacy and suggests concrete strategies to achieve that end. Recognizing that addressing health literacy requires more than behavior change on the part of dedicated professionals, a 2012 paper published by the Institute of Medicine (IOM) makes it clear that health care organizations have to tackle system-level factors to ensure that consumers can easily access health care and make informed decisions. ${ }^{3}$ The paper identifies ten attributes of "health literate organizations," that is, organizations that make it easier for people to navigate, understand, and use information and services to take care of their health. Table 1 lists the ten attributes and provides examples of each.

Published online October 11, 2013
In aggregate, these attributes focus on fostering a positive environment that enables people to access and benefit optimally from health care services.

Attribute 6-using health literacy strategies in interpersonal communication and confirming understanding-has the most direct relevance to physicians. Physicians exposed to health literacy training will recognize the references to strategies to confirm patient understanding, to limit the amount of information introduced in any single conversation, to avoid use of jargon and to overcome language barriers. Other aspects of this attribute include not making assumptions about an individual's prior health knowledge, skills or beliefs; listening actively and reflectively, without interruption; speaking clearly and at a moderate pace; encouraging question-asking; using graphics to supplement verbal and written explanations; and focusing on actionable information. Physicians can also play an active role in implementing Attribute 8 by selecting patient education materials that are easy to understand and act upon, including their provision in languages other than English for patients with limited English proficiency.

Physicians' responsibilities to address health literacy, however, are not restricted to improving the clinical encounter. For health care organizations to become health literate, physicians must also be willing to serve as health literacy champions. Physicians are often in positions of leadership at multiple levels in health organizations, from the corporate level to the clinical service line to the individual health care team. They are uniquely able to direct and foster change for each of the ten attributes listed in Table 1. We need physicians to use this influence to promote the integration of health literacy into every aspect of strategic and operational planning, evaluation and quality improvement (Attribute 2). They should insist on health literacy training for every staff member in their organizations (Attribute 3) and on the system redesign needed to effectively serve patients of all health literacy levels (Attribute 5).

In their roles as organizational leaders and managers, physicians can install mechanisms to systematically obtain input from patients on how to improve services to them (Attribute 4) and ensure they get the assistance they need in obtaining health information navigating the health care 
Table 1. Physicians' Roles in a Health Literate Organization, by Attribute

Attributes of a health literate $\quad$ Examples of physician role
organization:

1. Has leadership that makes health literacy integral to its mission, structure and operations

2. Integrates health literacy into planning, evaluation measures, patient safety and quality improvement

3. Prepares the workforce to be health literate and monitors progress

4. Includes populations served in the design, implementation and evaluation of health information and services

5. Meets needs of populations with a range of health literacy skills while avoiding stigmatization

6. Uses health literacy strategies in interpersonal communications and confirms understanding at all points of contact

7. Provides easy access to health information and services and navigation assistance

8. Designs and distributes print, audio/visual materials, and social media content that is easy to understand and act on

9. Addresses health literacy in high-risk situations, including care transitions and communications about medicines

10. Communicates clearly what health plans cover and what individuals will have to pay for services
- Become a physician champion for health literacy

- Make clear and effective communication a priority

- Support system and space redesign

- Join state or community health literacy coalitions

- Participate in health literacy

research to expand evidence base

- Conduct health literacy organizational assessments

- Assess the impact of policies and programs on individuals with limited health literacy

- Factor health literacy into all patient safety plans

- Hire diverse staff with expertise in health literacy

- Set goals for training of staff at all levels

- Include individuals who are adult learners and/or have limited health literacy information and services from individuals who use them

- Adopt health literacy universal precautions, such as offering everyone help with health literacy tasks

- Allocate resources proportionate to the with limited health literacy

- Confirm understanding (e.g. using the Teach-Back, Show-Me or Chunk-and-Check methods)

- Secure language assistance for speakers of languages other than English

- Limit to 2-3 messages at a time

- Use easily understood symbols in way-finding signage

- Make electronic patient portals user-centered and provide training on how to use them

- Facilitate scheduling appointments with other services.

- Involve diverse audiences, including those with limited health literacy, in development

- Use a quality translation process to produce materials in languages other than English

- Prioritize high-risk situations (e.g., informed consent for surgery and other invasive procedures

- Emphasize high-risk topics (e.g., conditions that require extensive self-management)

- Provide easy-to-understand descriptions of health insurance policies

- Communicate the out-ofpocket costs for health care services before they are delivered
- Obtain feedback on health concentration of individuals and rigorous user testing system (Attribute 7). Physician leaders allocate resources among competing demands and therefore can address Attribute 9 by directing resources to where the misunderstandings would have the most severe consequences. Finally, physician leaders can promote price transparency in their organizations, making communication about the out-of-pocket cost of services both easy to understand and timely (Attribute 10).

Fortunately, there are a number of tools available to assist physicians in helping their organizations become health literate, several of which are listed in the IOM paper. ${ }^{3}$ To enhance their own health literacy skills, physicians can make use of free online training. Other freely available resources include organizational health literacy assessments that identify strengths and opportunities for improvement for physician practices, clinics and hospitals. These tools range from informal self assessments to rigorous surveys of patients and/or staff. Completing an assessment at regular intervals can be part of a measurement program to chart organizational progress in promoting health literacy.

Being a health literate organization should not be seen as a luxury, something that is addressed after more "critical" elements of health care have been implemented. Rather, it is a necessary prerequisite to assuring patient safety, promoting adherence, enhancing self-efficacy and improving patient outcomes. Individuals with limited health literacy experience more serious medication errors, higher rates of emergency room visits and hospitalizations, worse preventive care and health outcomes for their children, and increased mortality compared with individuals with adequate health literacy. ${ }^{4} \mathrm{~A}$ health literate organization recognizes that virtually everyone faces health literacy challenges at some time or other, and that everyone benefits from clear communication. A health literate health care organization therefore institutes health literacy "universal precautions" - assuming that every patient is at risk of misunderstanding. The Health Literacy Universal Toolkit ${ }^{5}$ can help physicians lead their practices in implementing health literacy universal precautions. Professional organizations, such as the American Academy of Pediatrics, have also developed manuals and resources to assist physicians in health literate communications with all of their patients.

Without policies and infrastructure to support them, even the most conscientious physicians cannot single-handedly provide truly health literate care to their patients. There is no time in busy clinicians' schedules to add yet another set of unreimbursed activities. Being health literate must be a new way of delivering care rather than an add-on. For national health literacy goals to be met, health care organizations must "hard wire" health literacy into their standard operating procedures. During this period of health care transformation, physicians have a crucial role to play in realizing the promise of a health literate health care organization. 


\section{REFERENCES}

Acknowledgments: Dr. Schillinger was supported, in part, by European Commission Seventh Framework Programme's "Diabetes Literacy" Study.

Conflict of Interest: The authors declare that they do not have a conflicts of interest.

Corresponding Author: Dean Schillinger, MD; Division of General Internal Medicine, Department of Medicine University of California, San Francisco, UCSF Box 1364, San Francisco, CA 94143-1364, USA (e-mail: dschillinger@medsfgh.ucsf.edcu).
1. Kutner M, Greenberg E, Jin Y, Paulsen C. The health literacy of America's adults: results from the 2003 National Assessment of Adult Literacy. Washington DC: National Center for Educational Statistics; 2006 (NCES publication no. 2006-483).

2. U.S. Department of Health and Human Services Office of Disease Prevention and Health Promotion. National action plan to improve health literacy. Washington, DC; 2010.

3. Brach C, Keller D, Hernandez LM, et al. Ten attributes of a health literate health care organization. Washington DC: Institute of Medicine; 2012.

4. Berkman ND, Sheridan SL, Donahue KE, Halpern DJ, Crotty K. Low health literacy and health outcomes: an updated systematic review. Ann Intern Med. 2011;155:97-107.

5. DeWalt DA, Callahan LF, Hawk VH, et al. Health literacy universal precautions toolkit. Rockville: Agency for Healthcare Research and Quality; 2010 (AHRQ publication no. 10-0046-EF). 